\title{
Salvage of Failed Femoral Neck Fracture Fixation with Conversion Total Hip Arthroplasty Using the Direct Anterior Approach
}

\author{
Andrew Yun, MD, MBA, Marilena Qutami, PA-C, MMSc, Kory B. Dylan Pasko, BS \\ Department of Orthopedic Surgery, Center for Hip and Knee Replacement, \\ Providence Saint John's Health Center, Santa Monica, CA, USA
}

Purpose: Failed femoral neck fracture (FNF) fixation with in situ pinning presents a surgical challenge. Osteoporotic bone, retained hardware, and a typically elderly population magnify the risks of surgery. Here, outcomes of conversion total hip arthroplasty (THA) using two separate incisions in these high-risk patients were examined.

Materials and Methods: Medical records for 42 patients with a prior history of FNF fixation who underwent conversion THA with hardware removal between 2009 and 2019 were retrospectively reviewed. Surgery was performed by a single surgeon at a single institution. All patients underwent hardware removal followed by direct anterior approach (DAA) THA using two separate incisions. Clinical outcomes, radiographic findings, and perioperative morbidity and mortality are reported.

Results: Clinically, there were no postoperative dislocations, periprosthetic fractures, or infections at follow-up. After a mean follow-up of 4 years, the mean hip disability and osteoarthritis outcome score, junior (HOOS, Jr) was 91. Radiographically, the mean postoperative cup abduction was 44 degrees and the mean cup anteversion was 21 degrees with an improvement in preoperative leg length discrepancy. Perioperative complications included one case of immediate foot drop and two readmissions for medical issues. One patient died one month after conversion THA.

Conclusion: Salvage of failed FNF treatment may be managed with conversion THA and DAA with a separate incision for hardware removal. Preservation of posterior soft tissues using a DAA and intraoperative fluoroscopy may mitigate well-known complications related to fracture and dislocation. While favorable clinical outcomes are possible, salvage surgery is still not without substantial surgical and medical risks.

Key Words: Total hip arthroplasty, Femoral neck fracture, Posttraumatic arthritis, Hardware removal, Direct anterior approach

Submitted: May 22, 2020 1st revision: June 3, 2020

Final acceptance: July 13, 2020

Address reprint request to

Kory B. Dylan Pasko, BS

(https://orcid.org/0000-0001-8669-344X)

Department of Orthopedic Surgery, Center for Hip and Knee

Replacement, Providence Saint John's Health Center, 2121 Santa

Monica Blvd, Santa Monica, CA 90404, USA

TEL: +1-310-582-7474 FAX: +1-310-582-7481

E-mail: Korypaskodg.ucla.edu
This is an Open Access article distributed under the terms of the Creative Commons Attribution Non-Commercial License (http://creativecommons. org/licenses/by-nc/4.0) which permits unrestricted non-commercial use, distribution, and reproduction in any medium, provided the original work is properly cited. 


\section{Hip \& Pelvis}

Hip Pelvis 32(4): 199-206, 2020

\section{INTRODUCTION}

Failure of femoral neck fixation for intracapsular hip fractures occurs in up to $10 \%$ of patients ${ }^{1}$. Aseptic complications include nonunion, malunion, osteonecrosis, and post-traumatic arthritis (Fig. 1). Unfortunately, conversion total hip arthroplasty (THA) after failed treatment is a salvage procedure that carries a historically greater risk of fracture and dislocation compared to primary $\mathrm{THA}^{2-4}$.

Weak and osteoporotic bone, retained hardware, and a fragile elderly population compound the risks of surgery after prior closed reduction and pinning ${ }^{2}$. Standard technical recommendations (e.g., use of longer stems, cement fixation, extensile approaches) have not reliably reduced the rate of worse outcomes. Consequently, conversion THA has been found to be more comparable to a revision THA in terms of blood loss, rate of dislocation, length of stay, and resource utilization ${ }^{2}$.

An alternative technique was explored to potentially minimize these risks. In this study, a conversion using a direct anterior approach (DAA) THA with two incisions was conducted and long-term outcomes, perioperative course and complications were examined.

\section{MATERIALS AND METHODS}

A total of 42 consecutive patients with failed in situ pinning of femoral neck fractures (FNF) who required con- version THA between 2009 and 2019 were retrospectively reviewed. Only patients with prior treatment of intracapsular hip fractures treated with pins were included; patients with extracapsular or intertrochanteric hip fractures were excluded. For the patients in this series, a small initial incision was made laterally through the prior pin incision for screw removal. The wound was closed, and then conversion THA was performed by a DAA through a second incision. Surgery was performed by a single surgeon at a single institution with a minimum of 1-year follow-up. Charts were reviewed for indications, medical history, and mode of FNF treatment failure. The primary outcome measures were radiographic findings and the acute perioperative complications of fracture and dislocation. Secondary outcomes measured were patient reported hip disability and osteoarthritis outcome score, junior (HOOS, Jr), at follow-up, hospital course, infection, and death ${ }^{5}$. The study was approved by the Institutional Review Board (STUDY2020000184).

\section{Surgical Technique}

Patients were placed on the anterior hip replacement table (Hana; Mizuho, Union City, CA, USA) in the standard fashion. The leg was prepped to include the original lateral incision used for pinning. A 3-cm incision was made through the old incision with dissection taken past the iliotibial band and vastus laterals to the level of the screws. Screws
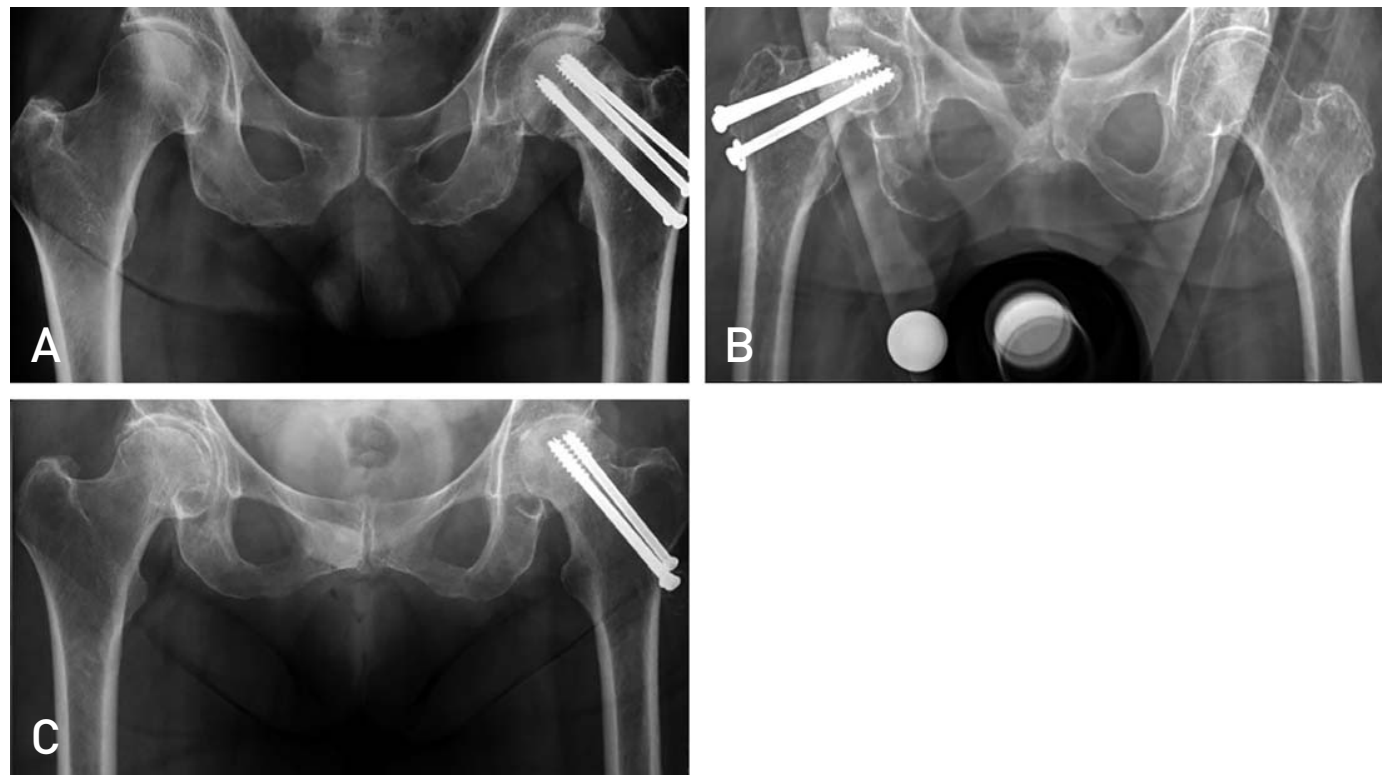

Fig. 1. Failed femoral neck fracture fixation requiring conversion total hip arthroplasty. (A) Anteroposterior (AP) radiograph with femoral neck nonunion 9 months after in situ pinning of left hip fracture. (B) AP radiograph with right hip osteonecrosis after pinning. Cortical collapse is visible. (C) AP radiograph of left hip post traumatic arthritis 6 years after pinning. 


\section{Hip \& Pelvis}

Andrew Yun et al. Salvage of Failed FNF Fixation with Conversion THA using DAA
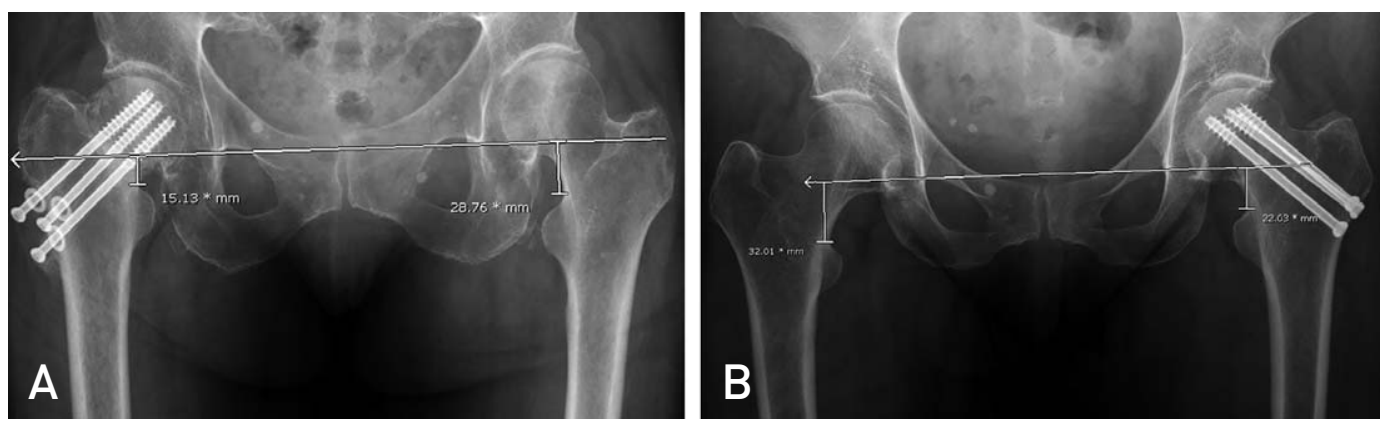

Fig. 2. Radiographic measurement of leg length discrepancy. (A) Anteroposterior (AP) radiograph with $14 \mathrm{~mm}$ of shortening in right hip and nonunion. (B) AP radiograph with $10 \mathrm{~mm}$ of shortening in left hip and malunion.

were removed by hand and with power tools. Fluoroscopy was used if there was difficulty locating the hardware. The wound was closed in layers and an occlusive dressing was placed over the closed incision.

The table was leveled, and attention was turned to the hip. In the method described by Matta et al. ${ }^{6)}$, an anterior approach was used with a Hana table, fluoroscopy, and digital radiography. After an $\mathrm{H}$-shaped capsulotomy with preservation of all capsular tissue, the head was drilled with a corkscrew to gain control of the proximal femur. With the boot unlocked, the femoral head was dislocated through rotation and traction on the head itself with the corkscrew. Importantly, minimal to no torsional stress was placed through the femoral shaft. A provisional osteotomy was made based on preoperative and intraoperative templating.

A cementless, titanium, hemispherical, acetabular shell Reflection 3 (Smith \& Nephew, London, UK) prior to 2018; Trident II (Stryker, Kalamazoo, MI, USA) from 2018, was used with a neutral, highly cross-linked, fixed bearing polyethylene liner to accommodate the largest femoral head. On the femoral side, a standard length cementless titanium stem using a triple taper hydroxyapatite-coated design Corail (DePuy Synthes, Warsaw, IN, USA) was used for all patients. Intraoperative imaging was used to guide component position and sizing as well as hip length and offset. The capsule was completely repaired by formally reapproximating the capsular leaves at the end of the procedure.

In this series, patients were allowed to progress with weight bearing as tolerated and without dislocation precautions. Patients were anticoagulated with aspirin for 3 weeks unless stronger anticoagulation was specifically indicated based on risk stratification, degree of immobility, and/or prior medical history.
Table 1. Demographic Information

\begin{tabular}{lc}
\hline \hline Measurement & Value \\
\hline No. of cases & 42 \\
Age (yr) & $71 \pm 12.6(44-89)$ \\
Sex & $14(33.3)$ \\
$\quad$ Male & $28(66.7)$ \\
$\quad$ Female & $23.8 \pm 4.2(18-36.2)$ \\
Body mass index $\left(\mathrm{kg} / \mathrm{m}^{2}\right)$ & $24(57.1)$ \\
Laterality & $18(42.9)$ \\
$\quad$ Right & \\
Left &
\end{tabular}

Values are presented as number only, mean \pm standard deviation (range), or number (\%).

\section{Radiographic Measurement}

Postoperatively, radiographs were evaluated for cup orientation and leg length discrepancy. Leg length discrepancy was determined by the vertical distance from a similar point on the lesser trochanters to a horizontal line drawn across the bottom of each radiographic teardrop (Fig. 2). Anteversion was calculated using the angle of the opening of the ellipse as calculated by the Radlink software (Radlink Inc., El Segundo, CA, USA). At follow-up, implants were evaluated for loosening, migration, and radiolucent lines.

\section{RESULTS}

The mean age of patients at the time of index surgery was 71 years (range: $44-89$ years). Patients included 28 female and 14 male. There were 24 right hips and 18 left hips. The mean body mass index at the time of index surgery was 23.8 $\mathrm{kg} / \mathrm{m}^{2}$ (range: $18-36.2 \mathrm{~kg} / \mathrm{m}^{2}$ ). Demographics are summarized in Table 1. The mean time from FNF to conversion THA was 11 years (range: $1-42$ years). The most common method of fixation was 3 screws (range: 1-4). The indications for 
conversion THA were as follows: 20 osteonecrosis (47.6\%), 11 post-traumatic arthritis (26.2\%), 7 malunions (16.7\%), and 4 nonunions $(9.5 \%)$. As seen in Table 2 , mean time from fracture to index surgery for each mode of failure was 9.5 years for osteonecrosis, 13.2 years for post-traumatic arthritis, 16.9 years for malunion, and 2.5 years for nonunion.

Postoperatively, the mean estimated blood loss was 245 $\mathrm{mL}$ (range: $75-650 \mathrm{~mL}$ ). Three patients required a transfusion $(7 \%)$ and no patients required care in the intensive care unit. The mean length of stay was 2 days (range: 1-7 days). As shown in Table 3, 36 patients were discharged to home, and 6 were discharged to a skilled nursing facility.

\section{Clinical Outcomes}

There were no known fractures or dislocations at a mean follow-up of 4 years (range: 1-10 years). At final followup, the average HOOS, Jr was 91 (range: 49-100). Due to the recent transition to patient reported outcome using the HOOS, Jr score, a preoperative score was not available for comparison. There was one reoperation in the immediate postoperative period; this patient was noted to have a postoperative foot drop and was immediately brought back to the operating room. On exploration, a large hematoma was evacuated. A postoperative magnetic resonance imaging revealed that the sciatic nerve was intact. After consultation with neurology, possible causes for the nerve palsy included compression from the hematoma or excessive traction on the sciatic nerve during femoral preparation. The foot drop did not resolve, and the patient was managed with an ankle foot orthosis. This patients' HOOS, Jr score was 85

Table 2. Mechanism of Failed Femoral Neck Fracture Fixation and Mean Time from Fracture to Index Surgery for Each Mode of Failure

\begin{tabular}{lc}
\hline \hline Variable & Value \\
\hline Preoperative diagnosis (no. of cases) & \\
Osteonecrosis & $20(47.6)$ \\
Post-traumatic arthritis & $11(26.2)$ \\
Malunion & $7(16.7)$ \\
Nonunion & $4(9.5)$ \\
Mean time from fracture to & \\
index surgery lyr) & \\
Osteonecrosis & $9.5 \pm 9.8$ \\
Post-traumatic arthritis & $13.2 \pm 16$ \\
Malunion & $16.9 \pm 15.1$ \\
Nonunion & $2.5 \pm 1.7$ \\
\hline
\end{tabular}

Values are presented as number $(\%)$ or mean \pm standard deviation. at 7 years follow-up. In terms of perioperative medical complications, there were no infections. One patient was readmitted 4 weeks following surgery with a small bowel obstruction and recovered after a course of bowel rest with a nasogastric tube. Another patient with a history of nephropathy developed an acute chronic renal insufficiency due to volume depletion requiring admission to an outside hospital 5 weeks later. One 89-year-old patient died of unclear etiology one month after index surgery at a skilled nursing facility.

At final follow-up, there were 5 deaths, one acute, as noted. The time to death after conversion THA for the other 4 patients was 2 to 6 years. The causes of death in the latter group were unrelated to conversion THA.

\section{Radiographic Outcomes}

For the acetabular implant, the mean abduction angle was 44 degrees (range: $42-50$ degrees) and the mean anteversion was 21 degrees (range: 16-26 degrees). In terms of leg length discrepancy, the mean amount of preoperative shortening was $7 \mathrm{~mm}$ (range: 0-19 $\mathrm{mm}$ ). The mean amount of operative limb lengthening was $5 \mathrm{~mm}$ (range: 0-19 $\mathrm{mm}$ ). Postoperatively, the mean limb length inequality was 2 $\mathrm{mm}$ (range: 0-11 mm). At final follow-up, all hip implants appeared stable without loosening or subsidence (Fig. 3). Radiographic results are summarized in Table 4.

\section{DISCUSSION}

Although the success of in situ pinning for FNFs remains high, there is a subset of patients who require further surgical management. Similar to the findings in the series described here, the reported incidence of complications

Table 3. Clinical Outcomes

\begin{tabular}{lc}
\hline \hline Measurement & Value \\
\hline Estimated blood loss at time & $245 \pm 178.1$ (75-650) \\
of surgery (mL) & $2 \pm 1.4(1-7)$ \\
Length of stay (day) & $36(85.7)$ \\
No. of patients discharged to & $6(14.3)$ \\
Home & $4 \pm 2.6(1-10)$ \\
Skilled nursing facility & $91 \pm 12.0(49-100)$ \\
Time to follow-up (yr) & \\
HOOS, Jr & \\
\hline Values are presented as mean \pm standard deviation (range) \\
or number (\%). \\
HOOS, Jr: hip disability and osteoarthritis disability score, \\
junior.
\end{tabular}




\section{Hip \& Pelvis}

Andrew Yun et al. Salvage of Failed FNF Fixation with Conversion THA using DAA
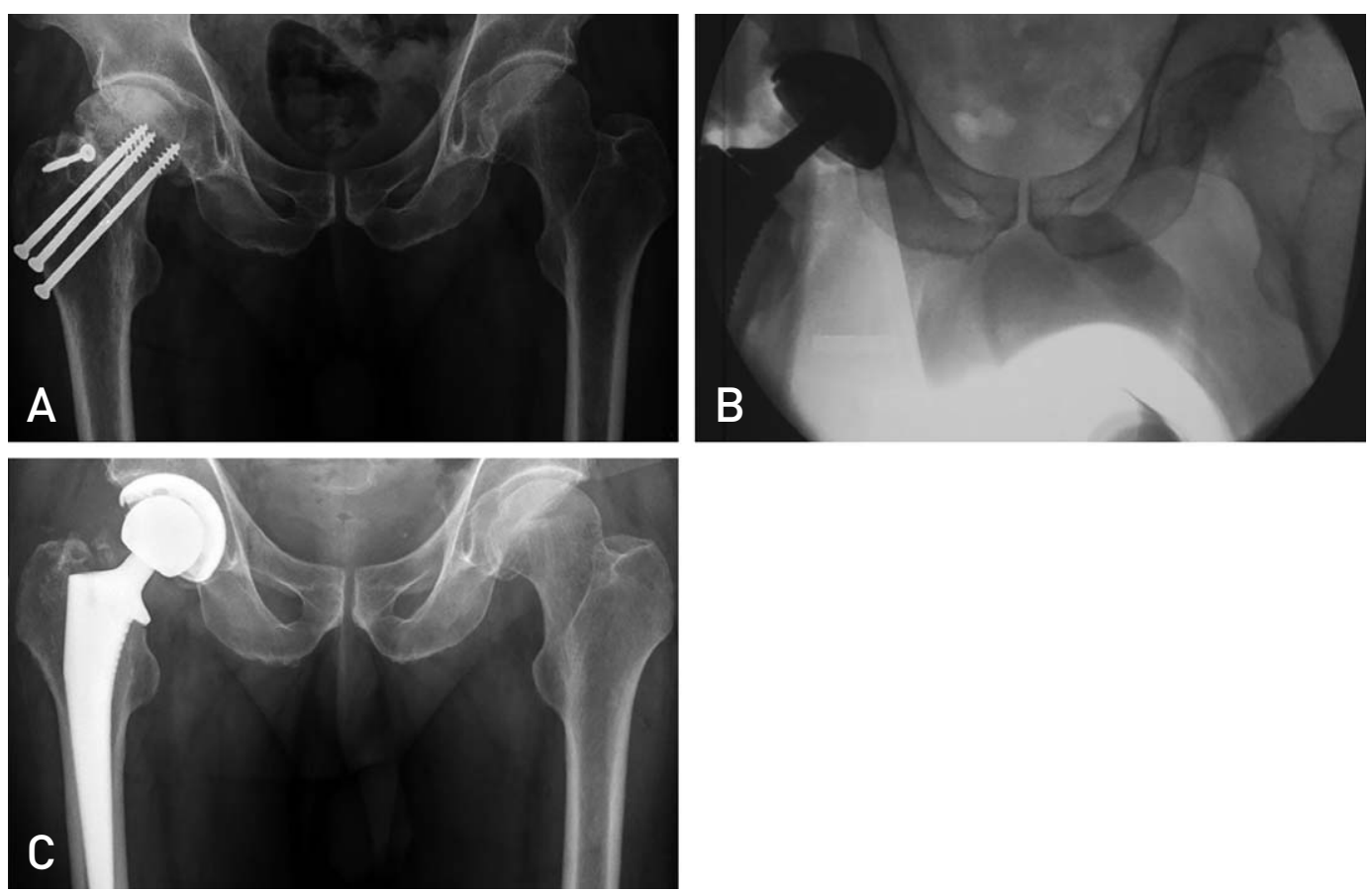

Fig. 3. Sequence of deformity and correction in conversion total hip arthroplasty. (A) Anteroposterior (AP) radiograph of failed treatment with posttraumatic arthritis and retained hardware. (B) Intraoperative imaging to assess implant position and orientation. (C) Follow-up AP radiograph at 4 years with well-fixed components.

Table 4. Pre- and Postoperative Radiographic Results

\begin{tabular}{lc}
\hline \hline Measurement & Value \\
\hline $\begin{array}{l}\text { Preoperative leg length discrepancy } \\
\text { (mm) }\end{array}$ & $7 \pm 5.3(0-19)$ \\
$\begin{array}{l}\text { Postoperative leg length discrepancy } \\
\text { (mm) }\end{array}$ & $2 \pm 2.5(0-11)$ \\
Acetabular cup abduction (degrees) & $44 \pm 2.6(42-50)$ \\
Acetabular cup anteversion (degrees) & $21 \pm 2.7(16-26)$ \\
\hline
\end{tabular}

Values are presented as mean \pm standard deviation (range).

after FNF treatment are as high as $44 \%$ for osteonecrosis, $35 \%$ for posttraumatic arthritis, and $21 \%$ for nonunion ${ }^{4}$. A database analysis of 9,962 patients with FNF's revealed that up to $10 \%$ of patients will fail treatment within a year ${ }^{1}$. Interestingly, the time to failure after FNF correlated with diagnosis in our series. Hence, patients with osteonecrosis and nonunion experienced early failure at a mean of 9.5 and 2.5 years, respectively; whereas patient with post-traumatic arthritis and malunion experienced late failure at a mean of 13.2 and 16.9 years.

Patients similar to those described here often complain of intractable pain, weakness, limp, and a shortened leg. Damage to either the femoral head or neck is too extensive for pin removal alone. Therefore, conversion THA becomes an appropriate salvage option.
Conversion THA after failure of in situ pinning, however, is fraught with a greater risk of surgical complications. Two series reported a fracture rate of 3-5\% 3,7 . To protect against the risk of proximal fracture through the screw holes, these authors recommend a sequence of femoral head dislocation with the hardware in place for torsional stability, reduction of the hip, hardware removal, and then secondary dislocation. While certainly reasonable, we have also found that hardware can be safely removed prior to head dislocation as long as the torsional force on the femur is controlled through the femoral head. Additionally, the concern of stress risers from the screw holes has also led some authors to suggest further protective measures. Hernandez et al. ${ }^{4)}$ recommended bone grafting the screw holes. In the series described here, the compaction broaching technique for the triple taper stem may have autografted the residual screw holes. Chen and Hozack ${ }^{8}$ has suggested that the screw holes should be bypassed by $3 \mathrm{~cm}$ with use of revision-style femoral implants. Interestingly, it was noted here that standard length implants performed as a reasonable alternative without any increased incidence of postoperative periprosthetic fractures. Careful examination of the radiographs reveals that the pins are typically placed in the thin cortex of metaphyseal bone at or above the level of the lesser trochanter, and standard-length stems easily bypass these 


\section{Hip \& Pelvis}

Hip Pelvis 32(4): 199-206, 2020
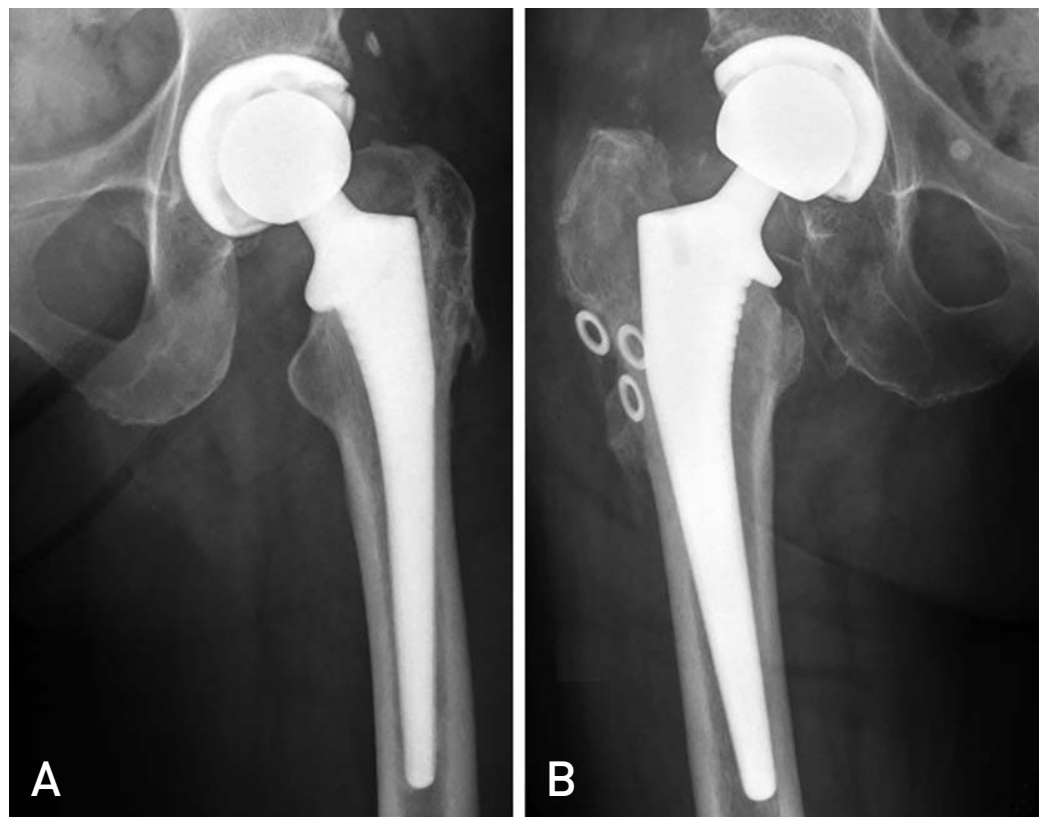

Fig. 4. Standard length femoral components easily bypass screw holes. (A) Close-up anteroposterior (AP) view of left hip shows stem bypassing screw holes noted by area of bone overgrowth. (B) Close-up AP view of right hip shows stem bypassing screw holes marked by retained washers. Because each screwhead had loosened from the bone beyond $1 \mathrm{~cm}$, no further dissection to remove the washers was done.
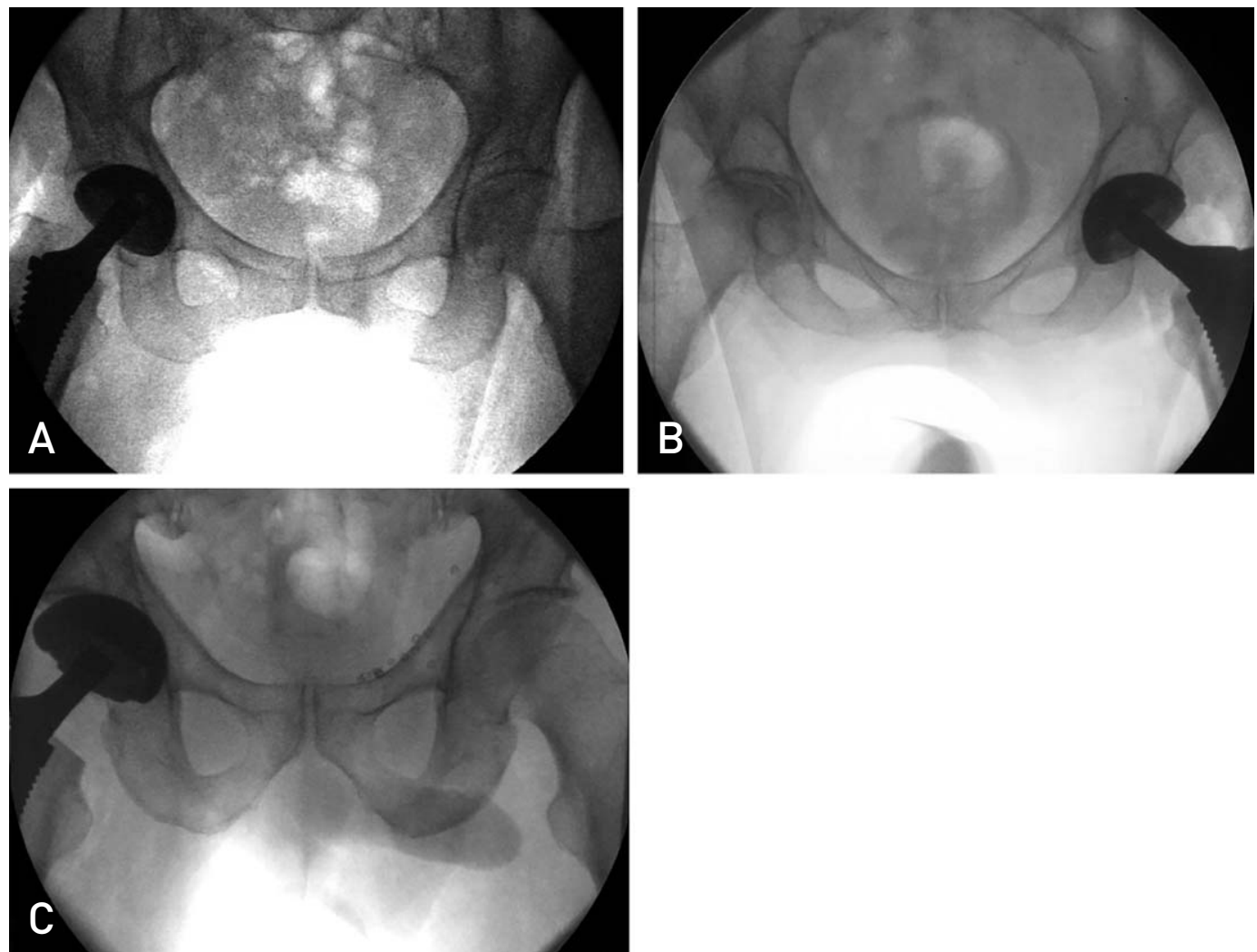

Fig. 5. Intraoperative imaging aided implant positioning. (A) Fluoroscopy of right hip conversion. Note cup orientation. (B) Fluoroscopy of left hip conversion. Note hip offset symmetry. (C) Fluoroscopy of right hip conversion. Note hip length symmetry. 
Andrew Yun et al. Salvage of Failed FNF Fixation with Conversion THA using DAA

proximal screw holes by at least $3 \mathrm{~cm}$ (Fig. 4). Unlike diaphyseal holes that remain after dynamic hip screws or short cephalomedullary nails, the more superior metaphyseal holes from in situ pinning may be less susceptible to fracture overall, as also theorized by Hernandez et al. ${ }^{4)}$ and Mortazavi et al. ${ }^{9}$.

Postoperative dislocation rates of up to $20 \%$ represent another potential complication of conversion after $\mathrm{FNF}^{10)}$. There is concern over associated damage to the soft tissue structures after a fall, and further surgery may further reduce the intrinsic stability around the hip. Given that prior publications have reported a dislocation rate of 5-20\% with the posterior approach, it has been recommended that hemiarthroplasty or a dual mobility construct be used to augment stability in high risk patients ${ }^{3,4}$. In the group described here, however, fixed-bearing constructs with a large head were chosen instead. Our series revealed no postoperative dislocations and it was noted that the DAA window did not require an extensile dissection and preserved the posterior soft tissues. A formal capsular repair rather than capsulectomy also augmented postoperative stability. It is possible that the absence of dislocations may be partly related to avoiding dissection of the posterior capsule associated with an anterior approach. Similarly, Mortazavi et al. ${ }^{9}$ reduced the dislocation rate to $0 \%$ using an anterolateral approach further demonstrating that maintaining the posterior soft tissues may play an important role in decreasing dislocation risk in this vulnerable cohort. Additionally, we found the use of intraoperative imaging for cup orientation, implant sizing, and hip length helps minimize the risk of outliers that may contribute to the risk of dislocation (Fig. 5).

The underlying health, cognitive decline, and overall fragility of these patients is also of concern. Hernandez et al. ${ }^{4}$ noted a mortality rate of $23 \%$ at two years after conversion. Although the average age of patients in our series was 71 years, eight were 80 years or older at time of conversion THA. Many had significant comorbidities typical of this age group. Two patients required acute rehospitalization for medical issues, and one died shortly after surgery. Fortunately, the recovery of the remaining patients after conversion THA was otherwise uneventful. Although most of the patients described here recovered without major incident, we agree that conversion THA carries an increased risk of complications and morbidity and that there is a clear concern for greater resource utilization, as warned by Schwarzkopf et al. ${ }^{2)}$.

The major limitation of this study is that it is a small retrospective series describing the experience of a single sur- geon. With a single surgeon there is an intrinsic risk of bias, so the aim of this analysis was to focus on objective points of reference (e.g., complications and radiographic outcomes). With the limitation of only 42 patients, a larger sample population may reveal a complication profile similar to that of other series. Another major limitation is the lack of a control group for comparison. A comparative cohort of patients treated with a posterior approach and intraoperative imaging could potentially demonstrate similarly favorable findings. Alternatively, future comparative studies with computer navigation or robotic assisted conversion THA may provide equivalent or even improved results compared to intraoperative fluoroscopy. Although a minimum 2-year follow-up is more common, we chose a minimum 1-year follow-up to include 4 additional patients for a greater pool for analysis, since the primary outcome measures were perioperative complications, fractures, and dislocations. Finally, as stated previously, a preoperative HOOS, Jr score was not available for comparison.

\section{CONCLUSION}

Failed FNF management remains perilous and technically challenging. Patients with osteonecrosis, nonunion, or secondary changes are substantially disabled and with limited options. While reasonable outcomes with conversion THA are possible, these salvage procedures are still not without substantial risk. The series described here is intended to highlight a potential alternative approach to conventional technical recommendations for these high-risk patients in the hope of minimizing future complications. Further investigation is warranted to reproduce these results.

\section{CONFLICT OF INTEREST}

The authors declare that there is no potential conflict of interest relevant to this article.

\section{REFERENCES}

1. Kahlenberg CA, Richardson SS, Schairer WW, Cross MB. Rates and risk factors of conversion hip arthroplasty after closed reduction percutaneous hip pinning for femoral neck fractures-a population analysis. J Arthroplasty. 2018;33: 771-6.

2. Schwarzkopf R, Manzano G, Woolwine S, Slover J. Salvage treatment of hip fractures after failure of surgical fixation: a systematic review. Orthop Knowl Online J. 2015;13. doi: 10.5435/OKOJ-13-3-3

3. Morice A, Ducellier F, Bizot P. Total hip arthroplasty after 


\section{Hip \& Pelvis}

Hip Pelvis 32(4): 199-206, 2020

failed fixation of a proximal femur fracture: analysis of 59 cases of intra- and extra-capsular fractures. Orthop Traumatol Surg Res. 2018;104:681-6.

4. Hernandez NM, Chalmers BP, Perry KI, Berry DJ, Yuan BJ, Abdel MP. Total hip arthroplasty after in situ fixation of minimally displaced femoral neck fractures in elderly patients. J Arthroplasty. 2018;33:144-8.

5. Lyman S, Lee YY, Franklin PD, Li W, Mayman DJ, Padgett DE. Validation of the HOOS, JR: a short-form hip replacement survey. Clin Orthop Relat Res. 2016;474:1472-82.

6. Matta JM, Shahrdar C, Ferguson T. Single-incision anterior approach for total hip arthroplasty on an orthopaedic table. Clin Orthop Relat Res. 2005;441:115-24.

7. Archibeck MJ, Carothers JT, Tripuraneni KR, White RE Jr.
Total hip arthroplasty after failed internal fixation of proximal femoral fractures. J Arthroplasty. 2013;28:168-71.

8. Chen AF, Hozack WJ. Component selection in revision total hip arthroplasty. Orthop Clin North Am. 2014;45:275-86.

9. Mortazavi SM, R Greenky M, Bican O, Kane P, Parvizi J, Hozack WJ. Total hip arthroplasty after prior surgical treatment of hip fracture is it always challenging? J Arthroplasty. 2012;27:31-6.

10. McKinley JC, Robinson CM. Treatment of displaced intracapsular hip fractures with total hip arthroplasty: comparison of primary arthroplasty with early salvage arthroplasty after failed internal fixation. J Bone Joint Surg Am. 2002; $84: 2010-5$. 